\title{
Trabecular bone recovers from mechanical unloading primarily by restoring its mechanical function rather than its morphology
}

\author{
Engin Ozcivici ${ }^{\mathrm{a}, \mathrm{b}, *}$, Stefan Judex ${ }^{\mathrm{b}}$ \\ a Department of Mechanical Engineering, Izmir Institute of Technology, Urla, Izmir, Turkey \\ b Department of Biomedical Engineering, Stony Brook University, Stony Brook, NY 11794, USA
}

\section{A R T I C L E I N F O}

\section{Article history:}

Received 8 October 2013

Revised 13 May 2014

Accepted 14 May 2014

Available online 21 May 2014

Edited by: Robert Recker

\section{Keywords:}

Mechanical loading

Stress

Recovery

Genetic research

Finite element method

\begin{abstract}
A B S T R A C T
Upon returning to normal ambulatory activities, the recovery of trabecular bone lost during unloading is limited. Here, using a mouse population that displayed a large range of skeletal susceptibility to unloading and reambulation, we tested the impact of changes in trabecular bone morphology during unloading and reambulation on its simulated mechanical properties. Female adult mice from a double cross of BALB/cByJ and $\mathrm{C} 3 \mathrm{H} / \mathrm{HeJ}$ strains $(\mathrm{n}=352)$ underwent $3 \mathrm{wk}$ of hindlimb unloading followed by $3 \mathrm{wk}$ of reambulation. Normally ambulating mice served as controls $(n=30)$. As quantified longitudinally by in vivo $\mu C T$, unloading led to an average loss of $43 \%$ of trabecular bone volume fraction (BV/TV) in the distal femur. Finite element models of the $\mu \mathrm{CT}$ tomographies showed that deterioration of the trabecular structure raised trabecular peak Von-Mises (PVM) stresses on average by $27 \%$, indicating a significant increase in the risk of mechanical failure compared to baseline. Further, skewness of the Von-Mises stress distributions (SVM) increased by $104 \%$ with unloading, indicating that the trabecular structure became inefficient in resisting the applied load. During reambulation, bone of experimental mice recovered on average only $10 \%$ of its lost BV/TV. Even though the addition of trabecular tissue was small during reambulation, PVM and SVM as indicators of risk of mechanical failure decreased by $56 \%$ and $57 \%$, respectively. Large individual differences in the response of trabecular bone, together with a large sample size, facilitated stratification of experimental mice based on the level of recovery. As a fraction of all mice, $66 \%$ of the population showed some degree of recovery in BV/TV while in $89 \%$ and $87 \%$ of all mice, PVM and SVM decreased during reambulation, respectively. At the end of the reambulation phase, only $8 \%$ of the population recovered half of the unloading induced losses in BV/TV while $50 \%$ and $49 \%$ of the population recovered half of the unloading induced deterioration in PVM and SVM, respectively. The association between morphological and mechanical variables was strong at baseline but progressively decreased during the unloading and reambulation cycles. The preferential recovery of trabecular micromechanical properties over bone volume fraction emphasizes that mechanical demand during reambulation does not, at least initially, seek to restore bone's morphology but its mechanical integrity.
\end{abstract}

(c) 2014 Elsevier Inc. All rights reserved.

\section{Introduction}

Loss of weight-bearing in skeletal extremities reduces tissue mass as mechanical loads provide critical anabolic and anti-catabolic signals [1-3]. The accompanying deterioration of bone's estimated [4] and actual [5] mechanical properties, combined with decreases in muscle strength [6,7] and postural stability [8] increase the risk of traumatic and non-traumatic fractures, particularly upon returning to regular weight bearing activities [9-11]. Daily bouts of exercise [12] or highfrequency motions [13-15] can partially alleviate the reduction in bone quantity, morphology and mechanical properties during

\footnotetext{
* Corresponding author at: Department of Mechanical Engineering, Rm 110, Izmir Institute of Technology, Urla, Izmir, 35430, Turkey. Fax: +902327506701.

E-mail address: enginozcivici@iyte.edu.tr (E. Ozcivici).
}

unloading. However, once mechanical loads are reintroduced to skeletal extremities during reambulation, recovery of bone mass is often slow and incomplete. If full recovery is accomplished, it requires several times the duration of unloading [16-18]. During the initial phase of reambulation, bone mass may even continue to deteriorate in humans $[16,19]$ and rodents [20], further compromising bone's structural integrity at a time when mechanical support is critical to withstand reloading of the skeleton.

To more accurately assess fracture risk of eroded skeletal sites than by dual X-ray absorptiometry (DXA) or quantitative computed tomography (QCT) alone, mechanical testing of bone can be performed non-invasively by simulating the application of mechanical loads to the imaged bone structure with the finite element (FE) method [21-24]. Simulations can be performed for specific sub-regions of the scanned images and filtered for different features. For instance, a digital structure from which non load-bearing trabeculae were digitally 
removed better predicts mechanical strength than a structure containing load-bearing and non load-bearing elements [25]. Further, the high spatial resolution of the FE method at the micron-scale can aid in understanding the risk of mechanical failure, and changes in it, through the detection of those locations in which peak stresses occur as well as by testing whether the microstructural arrangement of trabeculae allows for efficient load transfer without overstressing individual elements [26].

FE analysis of skeletal sites in astronauts indicated a $5 \%$ loss in proximal femoral strength per month [4], at least twice the rate previously suggested by methods based on bone density and mass [2,27,28]. A similar discrepancy between the loss of trabecular bone quantity and its mechanical properties has been observed in rodent unloading models $[14,29]$. As a result, a panel recently recommended the integration of FE analysis with QCT to more effectively evaluate astronaut bone health in future studies [3]. While FE analysis has been used to describe the deterioration of bone's mechanical properties during spaceflight and ground-based analogs, much less is known about changes in bone's mechanical properties during reambulation. After long-duration missions, QCT based estimates of bone strength suggested that recovery of bone mass was incongruent with recovery of its estimated mechanical properties [16]. While it is clear that during altered loading environments, bone's mechanical properties cannot be fully predicted from its morphology, little is known of how bone's simulated mechanical properties change across individuals with distinct mechano-sensitivity and which trabecular architectural variable(s) play a dominant role in modulating bone mechanics.

To address this question, we quantified longitudinal changes in simulated mechanical parameters of trabecular bone during unloading and reambulation using a 2nd generation (F2) genetically heterogeneous mouse population bred for producing a large range of responses to altered mechanical demand. For this experiment, the association of specific chromosomal regions with the magnitude of trabecular deterioration/recovery during unloading/reambulation has been reported for morphology [30] and estimated mechanical properties [31]. Here, we asked the following questions: (1) Are changes in simulated mechanical properties similar in magnitude and variability to morphologic changes during unloading and reambulation? (2) To what extent do architectural and mechanical traits recover in trabecular bone during reambulation across a population with a large range of mechano-responses? (3) Which architectural variables determine changes in bone's simulated mechanical properties during unloading/ reambulation?

\section{Materials and methods}

\section{Experimental design}

All procedures were reviewed and approved by the Institutional Animal Care and Use Committee (IACUC) of Stony Brook University. A total of 352 female adult (16 wk old) mice from a 2nd generation (F2) cross of BALB/CByJ (BALB - high response to mechanical unloading) and $\mathrm{C} 3 \mathrm{H} / \mathrm{HeJ}(\mathrm{C} 3 \mathrm{H}$ - low response to mechanical unloading) inbred strains were used [32]. At baseline $(n=436)$, mice were $\mu$ CT-scanned in vivo (vivaCT40, Scanco Medical, Switzerland) at the distal femoral metaphysis under isoflurane inhalation. Immediately following the scans, both hindlimbs were unloaded for $3 \mathrm{wk}$ [33]. Upon completing the unloading phase, $\mu \mathrm{CT}$ scans were taken $(\mathrm{n}=359)$ and mice were released for $3 \mathrm{wk}$ of normal cage activity (reambulation). Following reambulation $(\mathrm{n}=352)$, mice were $\mu \mathrm{CT}$ scanned again ( $22 \mathrm{wk}$ old $)$. Sample sizes were not identical at the three $\mu \mathrm{CT}$ scan time points primarily because of scheduling conflicts for the time consuming in vivo scans (mice that could not be scanned were sacrificed). A small number of mice did not adapt to hindlimb unloading and in accordance to the IACUC protocol, mice with deteriorating health and/or weight losses were terminated. Non-suspended age-matched control mice $(\mathrm{n}=30)$ were allowed regular cage activities throughout the 6 wk experimental period and received $\mu \mathrm{CT}$ scans at identical time points as experimental mice. Reconstructed $\mu \mathrm{CT}$ tomographies were directly converted into finite element (FE) models and subjected to uniaxial compression for the evaluation of trabecular micro-mechanical properties.

\section{Micro-computed tomography}

The distal femoral metaphysis was assessed at an isometric voxel size of $17.5 \mu \mathrm{m}$. The analyzed region comprised $1500 \mu \mathrm{m}$ (85 slices) with the most distal slice located $600 \mu \mathrm{m}$ (35 slices) proximal from the growth plate [32]. Trabecular bone was separated from cortical bone using an image processing language algorithm [34] followed by 3D Gaussian blurring with "sigma" and "support" values of 0.3 and 1 . The threshold value that segmented calcified tissue from background was set at $29.5 \%$ of the maximum voxel intensity for all mice and time points. For trabecular bone, bone volume fraction (BV/TV), connectedness (Conn.D), number (Tb.N) thickness (Tb.Th) and the degree of anisotropy (D.A) were determined.

\section{Finite element modeling}

Changes in mechanical properties induced by altered mechanical demand were evaluated in the femoral metaphysis (as defined above) comprising both trabecular and cortical bone. Every voxel representing trabecular or cortical bone was directly translated to a FE brick element with an isotropic size of $17.5 \mu \mathrm{m}$ (ScancoFE, Scanco Medical). A frictionless compression test in the longitudinal (dominant habitual loading) direction was applied to all FE models [14]. Linear elastic properties were assigned to trabecular and cortical bone with an elastic modulus (E) of 25 GPa and Poisson's ratio (v) of 0.3 [35-37]. FE solutions were completed via an iterative FE-solver [38] with a force $(\mathrm{N})$ and displacement $(\mathrm{mm})$ tolerance of $1 \times 10^{-4}$ (ScancoFE, Scanco Medical). During post-processing, voxels pertaining to cortical bone were excluded and simulated mechanical properties were analyzed only for trabecular bone. Thus, in contrast to a test in which only a trabecular core is subjected to a simulated compression test, here, trabecular bone was compressed in a more physiologic manner with force transfer from both endplates as well as from trabecular struts connected to the cortical shell [14].

Apparent stiffness values for trabecular bone were calculated as the ratio of input force to resultant displacement. To simplify the stress representation while including all stress components arising in normal and shear directions, Von-Mises (VM) stresses, a derived stress value that is closely related to failure in trabecular bone [39], were calculated for all time points. For all models, calculated VM stress values were normalized to the axial boundary force magnitude to ensure that all models were subjected to a $1 \mathrm{~N}$ compressive force. To prevent local artifacts and singularities, peak VM (PVM) stresses were calculated as the 95th percentile of the VM stress distribution [14]. Trabecular bone of F2 mice that showed higher PVM values under the same loading conditions was assumed to be more prone to mechanical failure.

Skewness of the VM stress distribution (SVM) was calculated for each individual's VM stress histograms to quantify the non-uniformity of the stress histogram as an indicator of trabecular efficiency in load bearing [26]. When the VM stress histogram is normally distributed, stress transfer is assumed to be efficient as most of the trabecular elements are subject to intermediate stresses. As the trabecular structure changes its morphology, the stress histogram may change its distribution. For instance, a left leaning distribution with a long tail to its right indicates a mechanically less efficient structure because the bulk of the trabecular elements is subjected to lower stresses while a relatively small number of elements on the right tail become disproportionally overstressed to support the applied load (Fig. 1). 


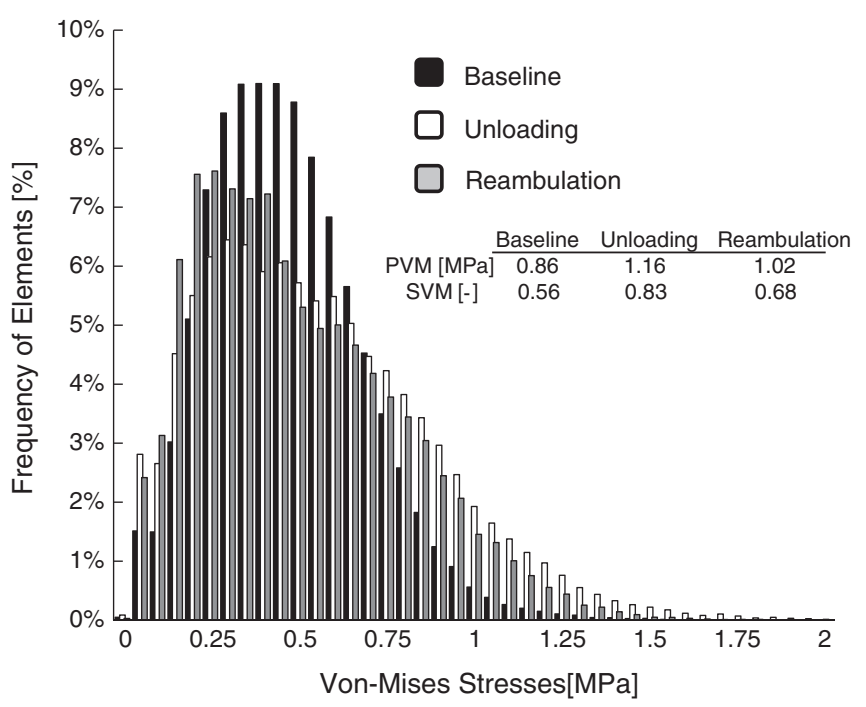

Fig. 1. Histograms of trabecular Von-Mises stresses normalized to number of FE elements of the trabecular distal femur in a single experimental mouse. $\mu \mathrm{CT}$ scans were taken at baseline, upon completion of unloading (wk 3), and upon completion of reambulation (wk 6) and directly translated into FE models. A simulated compression test was applied in the longitudinal direction. At baseline, stress values had a distribution that approached normality (black bars) but unloading induced deterioration of trabecular bone, increased peak stresses and skewed the stress distribution (white bars). Reambulation partially mitigated unloading induced changes in stress values and skewness (gray bars). Values for peak Von-Mises (PVM) and skewness of the Von-Mises stress distributions (SVM) in this representative example are shown in the nested table. Data from a single experimental mouse, rather than the average of the entire population, was used for ease of representation that is free of variations across individual mice.

\section{Statistics}

All data were presented as mean ( \pm standard deviation) and statistical significance was set at $5 \%$. Normality of data distributions was evaluated by fitting Gaussian curves to histograms and calculating $\mathrm{R}^{2}$ values. Unpaired t-tests were used to contrast longitudinal (\%) changes of morphological and simulated micro-mechanical values between experimental and age-matched control mice. Paired t-tests tested whether longitudinal changes in experimental mice were significant by contrasting data from the $6 \mathrm{wk}$ or $3 \mathrm{wk}$ time point to the previous time point. Paired t-tests were also used to compare longitudinal changes between unloading and reambulation in experimental mice. Coefficients of variations (COV) determined the normalized degree of variability for a given variable and were calculated as the ratio of standard deviation to mean value. Coefficients of determination $\left(R^{2}\right)$ were used to determine associations between bone morphology and mechanical variables at baseline as well as for relative (\%) longitudinal differences in these variables during unloading and reambulation.

\section{Results}

\section{Unloading}

Changes in trabecular morphology during unloading/reambulation are presented, at least in part, elsewhere [30]. Briefly, baseline parameters of trabecular BV/TV, Conn.D, Tb.N and Tb.Th (Table 1) showed large variability, reflecting differences in genetic make-up within this mouse population. Three weeks of hindlimb unloading caused significant losses of trabecular bone morphology in the distal femoral metaphysis, amounting to an average individual loss of $43 \%$ in BV/TV (Fig. 2, Table S1), 38\% in Conn.D, $17 \%$ in Tb.N, $18 \%$ in Tb.Th and 5\% in D.A (Tables 2, S1). Based on the large differences in the mechanoresponses of the inbred progenitor strains, trabecular bone's response to mechanical unloading was also highly variable, as evidenced by high coefficients of variations (COV) for changes in BV/TV (0.39),
Table 1

Trabecular bone architecture and simulated mechanical variables presented as mean $\pm \mathrm{SD}$ (COV) for control and experimental mice at baseline (16 wk) as represented by trabecular bone volume fraction, trabecular connectedness, trabecular number, trabecular thickness, degree of anisotropy, trabecular stiffness, peak Von-Mises stresses, and skewness of Von-Mises stress distributions. There were no significant differences between control and experimental mice prior to starting the experiment.

\begin{tabular}{lcc}
\hline & Control $(\mathrm{n}=436)$ & Experimental $(\mathrm{n}=30)$ \\
\hline BV/TV $(-)$ & $0.23 \pm 0.08(36 \%)$ & $0.21 \pm 0.08(38 \%)$ \\
Conn.D $\left(1 / \mathrm{mm}^{3}\right)$ & $95 \pm 29(30 \%)$ & $87 \pm 32(37 \%)$ \\
Tb.N $(1 / \mathrm{mm})$ & $4.62 \pm 0.81(18 \%)$ & $4.35 \pm 0.84(19 \%)$ \\
Tb.Th $(\mu \mathrm{m})$ & $67.5 \pm 9.4(14 \%)$ & $65.2 \pm 8.2(13 \%)$ \\
D.A $(-)$ & $1.65 \pm 0.10(6 \%)$ & $1.67 \pm 0.12(7 \%)$ \\
Stiffness $(\mathrm{N} / \mathrm{mm})$ & $2660 \pm 930(35 \%)$ & $2413 \pm 926(39 \%)$ \\
PVM $(\mathrm{MPa})$ & $0.96 \pm 0.15(15 \%)$ & $1.02 \pm 0.19(18 \%)$ \\
SVM $(-)$ & $0.49 \pm 0.13(28 \%)$ & $0.52 \pm 0.21(40 \%)$ \\
\hline
\end{tabular}

Conn.D (0.64), Tb.N (0.43), Tb.Th (0.44) and D.A (0.73). During the same 3 wk period, trabecular morphology in controls did not change (Fig. 2, Table 2). For all variables, longitudinal changes were significantly different $(\mathrm{p}<0.05)$ between unloaded and control mice.

Unloading compromised trabecular bone's simulated mechanical properties to a lesser extent than its bone volume fraction; at the end of the unloading period, apparent trabecular stiffness was $42 \%$ lower and peak Von Mises (PVM) stresses were $27 \%$ greater than at baseline (all $\mathrm{p}<0.05$, Fig. 2, Table S2). The skewness of the Von Mises stress distribution (SVM), an index of trabecular efficiency of load bearing, increased significantly by $104 \%$. Similar to morphological variables, the mechanical response to unloading was highly variable across individuals with COV of 0.46 for stiffness, 0.54 for PVM, and 0.77 for SVM. Changes in apparent stiffness, PVM, and SVM of unloaded mice were significantly different from those in age-matched controls (Fig. 2). In controls, mechanical variables were not different between the beginning and the end of this $3 \mathrm{wk}$ period.

\section{Reambulation}

During reambulation (change between end of reambulation and end of unloading), the average increase in BV/TV of the distal femur was $10 \%$, accompanied by a $16 \%$ increase in Tb.Th (Fig. 2, Tables 2, S1). In contrast, Conn.D, Tb.N and D.A continued to deteriorate during reambulation with significant average reductions of $-18 \%,-8 \%$ and $-3 \%$ (Tables 2, S1). Trabecular bone's response to reambulation was more variable than during unloading with a COV of 2.23 for changes in BV/TV, 1.80 for Conn.D., 1.07 for Tb.N, and 1.98 for D.A. Tb.Th was the only variable for which the COV pertaining to longitudinal changes was similar during reambulation (0.58) and unloading. Similar to the first $3 \mathrm{wk}$ phase, trabecular changes in control mice, with the exception of Tb.N, were also not significant during the second 3 wk phase, but all changes in reambulating mice were different $(p<0.05)$ from those in controls (Fig. 2, Table 2). Morphological changes that occurred during reambulation were also different from those occurring during unloading (Table 2).

Across all experimental mice, the three indices of trabecular bone mechanics improved during reambulation; trabecular stiffness increased by $13 \%$ while PVM and SVM decreased by $10 \%$ and $27 \%$ (all $\mathrm{p}<0.05$, Fig. 2, Table S2). For reambulation, COV for changes in stiffness (2.38) was greater than for unloading but COV for PVM (0.78) and SVM (0.91) were similar between reambulation and unloading. Reflecting the small morphologic changes during reambulation, agematched controls had insignificant changes in stiffness (1\%), PVM (-3\%) and SVM (-7\%) during this phase (Fig. 2). Mechanical changes that occurred during reambulation were significantly different from those occurring during unloading and from those occurring in agematched controls (Table 2).

For all mice that showed a decline in BV/TV during unloading ( $n=348$ ), recovery of trabecular parameters as a fraction of unloading 
a

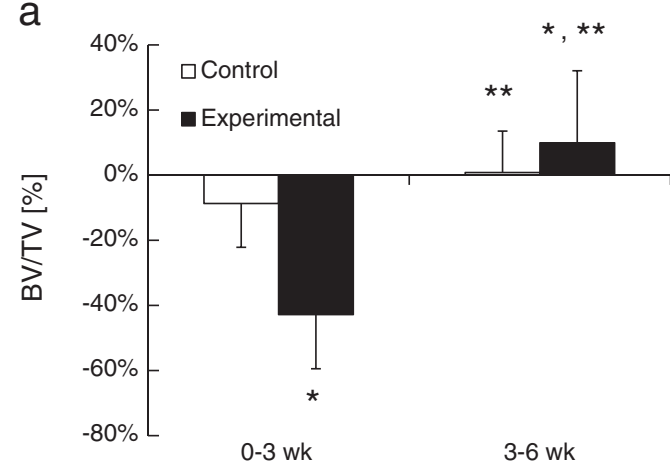

C

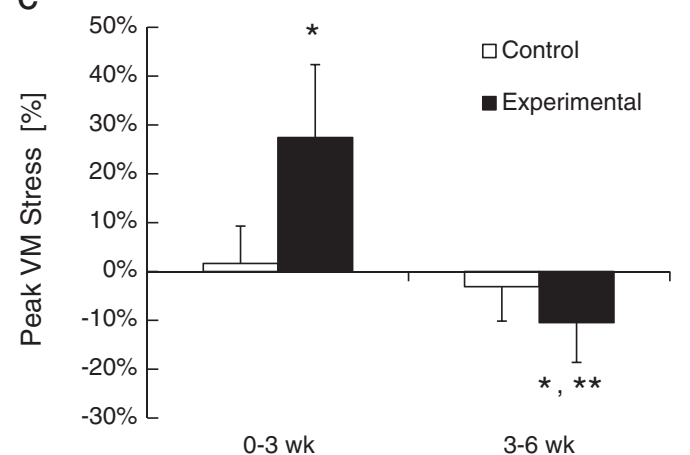

b

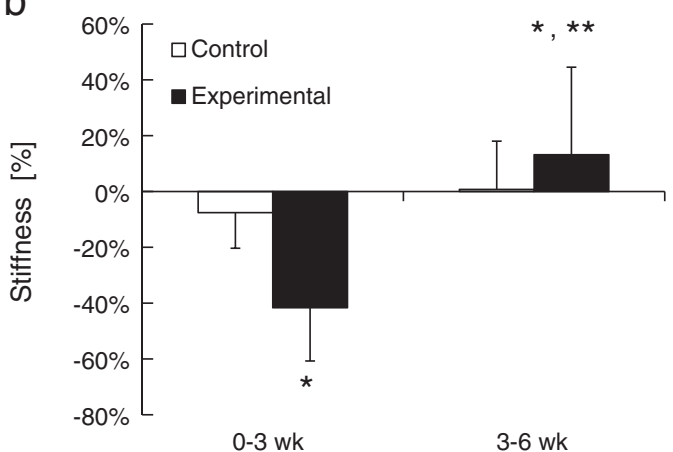

d

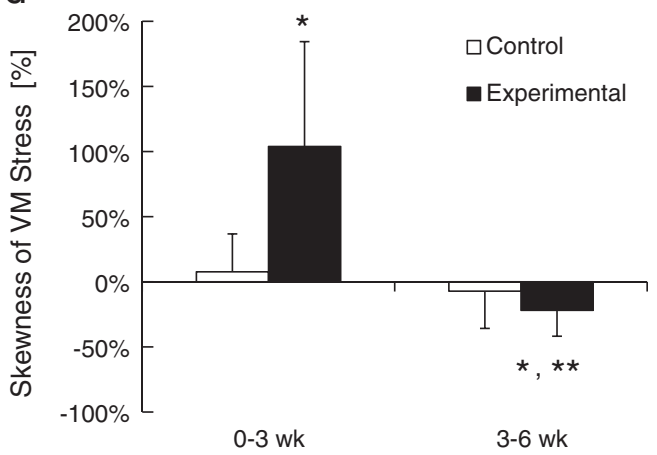

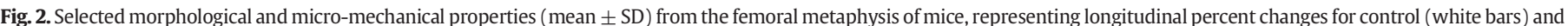

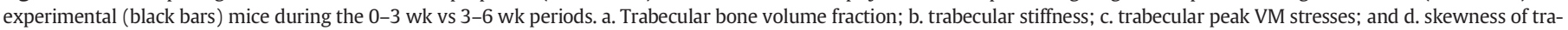

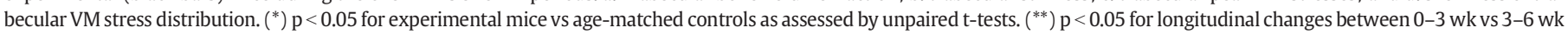
as assessed by paired t-tests.

induced deterioration was calculated for the reambulation phase. Only $66 \%$ of these mice were able to increase femoral BV/TV, demonstrating some level of recovery (Fig. 3). 9\% of mice recovered at least half of their losses (>50\%) in BV/TV while only $2 \%$ regained BV/TV to match baseline levels (>95\%). For Conn.D and Tb.N, $20 \%$ and 13\% of the population showed some recovery (Fig. 3). In contrast, $97 \%$ of mice showed positive recovery for Tb.Th. 76\% of mice recovered at least half of their losses while $22 \%$ achieved a recovery that was more than $95 \%$ (Fig. 3 ).

Recovery of trabecular stiffness for mice that showed negative changes for BV/TV during unloading was similar to the recovery of BV/ TV; $62 \%, 17 \%$ and $5 \%$ showed recovery of $>0 \%,>50 \%$ and $>95 \%$. Unlike stiffness, $91 \%$ of all mice showed positive recovery in PVM, 51\% recovered at least half of their losses and $12 \%$ experienced more than $95 \%$ recovery. Similarly, $87 \%$ of the mice showed recovery in SVM, 50\% recovered at least half of their losses, and 18\% experienced more than $95 \%$ recovery (Fig. 3 ).

\section{Table 2}

Individual longitudinal percent changes in trabecular bone architecture, represented by trabecular connectedness, number, thickness and degree of anisotropy between $0-3 \mathrm{wk}$ and 3-6 wk for control and unloaded mice. Percent changes were calculated for each individual mouse between two consecutive time points and then averaged across the population.

\begin{tabular}{|c|c|c|c|c|}
\hline & \multicolumn{2}{|l|}{$0-3 \mathrm{wk}$} & \multicolumn{2}{|l|}{ 3-6 wk } \\
\hline & Control & Experimental & Control & Experimental \\
\hline Conn.D & $-18 \pm 13 \%$ & $-38 \pm 24 \%^{*}$ & $-11 \pm 12 \%$ & $-18 \pm 32 \%^{* *}$ \\
\hline Tb.N & $-8 \pm 5 \%$ & $-17 \pm 7 \%$ & $-2 \pm 7 \%^{* *}$ & $-8 \pm 8 \%^{*}$ \\
\hline Tb.Th & $-1 \pm 6 \%$ & $-18 \pm 8 \%^{*}$ & $3 \pm 5 \%$ & $16 \pm 9 \%^{*}$ \\
\hline D.A & $-1 \pm 3 \%$ & $-5 \pm 4 \%^{*}$ & $0 \pm 3 \%$ & $-3 \pm 5 \%^{*}$ \\
\hline
\end{tabular}

$* \mathrm{p}<0.05$ for longitudinal (\%) changes between experimental mice and age-matched controls (unpaired t-test).

** $\mathrm{p}<0.05$ for longitudinal change between $0-3$ wk vs 3-6 wk (paired t-test).
Further analyses were performed on mice that did not recover BV/TV and instead exhibited further deterioration (FD) in BV/TV during reambulation ( $\mathrm{n}=117$ mice, $34 \%$ of the population). At baseline, the FD group had $13 \%$ higher BV/TV $(p<0.01$ ) compared to the positive recovery (PR) group. Other morphological variables were also greater in FD mice at baseline including Conn.D (13\%, p < 0.01), Tb.N ( $7 \%, \mathrm{p}<$ $0.01)$, and Tb.Th ( $3 \%, \mathrm{p}=0.03)$. No significant difference between FD and PR was observed in D.A. The difference in morphological indices between FD and PR mice at baseline extended to the simulated mechanical properties of trabecular bone, where stiffness was $17 \%$

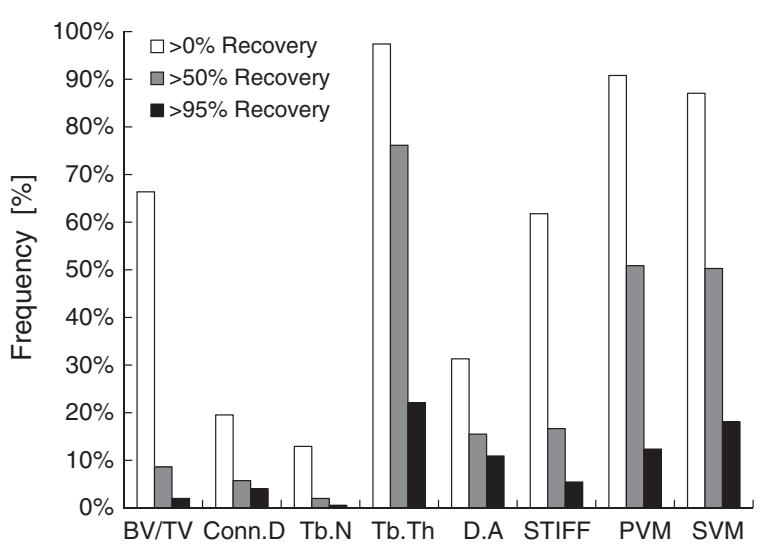

Fig. 3. Relative number of mice showing a given degree of recovery during reambulation. Only mice that experienced losses during unloading were included $(n=348)$. $>\mathbf{0} \%$ : Positive recovery during reambulation, $>\mathbf{5 0} \%$ : recovery of at least half of their losses, and $>$ 95\%: near complete recovery approaching baseline values. BV/TV: trabecular bone volume fraction; Conn.D: trabecular connectedness; Tb.Th: trabecular thickness; Tb.N: trabecular number; D.A: degree of anisotropy; STIFF: trabecular stiffness; PVM: peak VonMises stress; and SVM: skewness of Von-Mises stress histogram. 
Table 3

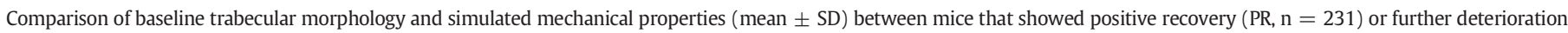
( $\mathrm{FD}, \mathrm{n}=117$ ) in BV/TV during reambulation.

\begin{tabular}{|c|c|c|c|c|}
\hline & $\mathrm{BV} / \mathrm{TV}(-)$ & Conn.D $\left(1 / \mathrm{mm}^{3}\right)$ & Tb.N $(1 / \mathrm{mm})$ & Tb.Th $(\mu \mathrm{m})$ \\
\hline Positive recovery (PR) & $0.20 \pm 0.08$ & $83 \pm 31$ & $4.25 \pm 0.81$ & $64.5 \pm 8.0$ \\
\hline \multirow[t]{2}{*}{ Further deterioration (FD) } & $0.23 \pm 0.08^{*}$ & $94 \pm 35^{*}$ & $4.53 \pm 0.87^{*}$ & $66.4 \pm 7.9^{*}$ \\
\hline & D.A $(-)$ & $\mathrm{STIFF}(\mathrm{N} / \mathrm{mm})$ & PVM (MPa) & $\operatorname{SVM}(-)$ \\
\hline Positive recovery (PR) & $1.66 \pm 0.11$ & $2249 \pm 848$ & $1.05 \pm 0.18$ & $0.55 \pm 0.21$ \\
\hline Further deterioration (FD) & $1.68 \pm 0.11$ & $2624 \pm 1012^{*}$ & $0.99 \pm 0.18^{*}$ & $0.50 \pm 0.19^{*}$ \\
\hline
\end{tabular}

* $\mathrm{p}<0.05$ between groups (unpaired t-test).

greater $(\mathrm{p}<0.01)$, PVM was $5 \%$ lower $(\mathrm{p}=0.01)$ and SVM was $9 \%$ lower $(p=0.03)$ in baseline FD mice compared to baseline PR mice (Table 3). Unlike the differences between FD and PR mice observed at baseline, longitudinal changes in morphological and simulated mechanical properties of trabecular bone during unloading did not differ between FD and PR mice (Table S3).

Of those mice that showed further deterioration in BV/TV during reambulation, only $9 \%$ and $1 \%$ showed some recovery of Conn.D and Tb.N respectively (Fig. 4). In spite of declining BV/TV, 26\% of this subpopulation exhibited recovery of trabecular stiffness; but only $4 \%$ were able to recover half of the losses in stiffness induced by unloading. Unlike the previous variables, $93 \%, 80 \%, 75 \%$ of this subpopulation demonstrated recovery in Tb.Th, PVM and SVM (Fig. 4). For Tb.Th, $44 \%$ of this subpopulation recovered half and $7 \%$ recovered more than $95 \%$ of unloading induced losses. Similarly, $24 \%$ and $32 \%$ of the subpopulation recovered more than half of the increases in PVM and SVM incurred during unloading even in the absence of any recovery for BV/TV (Fig. 4).

\section{Morphologic-mechanical associations}

Associations between BV/TV and simulated mechanical properties were strong at baseline, and became incrementally weaker when performed for changes incurred during unloading and reambulation (Table 4). Tb.N and Tb.Th, but not Conn.D and D.A, were strongly associated with trabecular mechanics at baseline (Table 4). During unloading and reambulation, Tb.Th and BV/TV were the architectural variables most highly linked to bone's mechanical properties (Table 4). Further correlations were performed on F2 subpopulations stratified for the

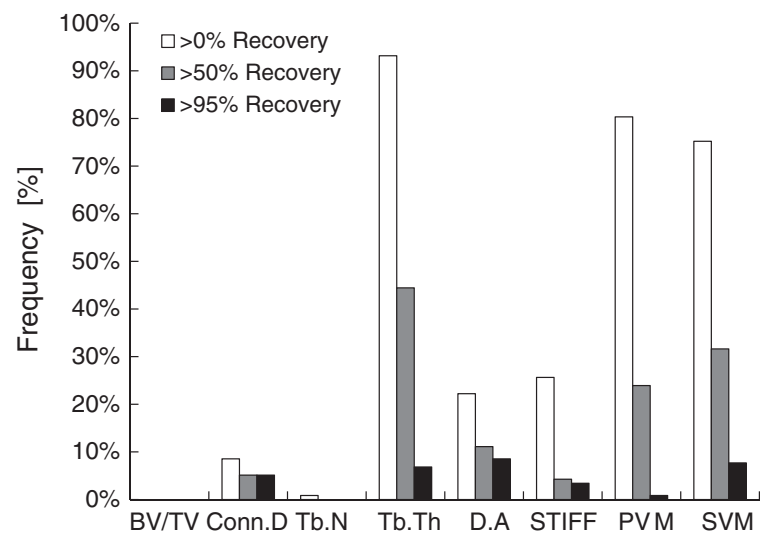

Fig. 4. Relative number of mice showing a given degree of recovery during reambulation. Only mice that did not show any recovery in BV/TV during reambulation were included $(n=117)$. $>\mathbf{0} \%$ : Positive recovery during reambulation, $>\mathbf{5 0} \%$ : recovery of at least half of their losses, and $>\mathbf{9 5} \%$ : near complete recovery approaching baseline values. BV/TV: trabecular bone volume fraction; Conn.D: trabecular connectedness; Tb.Th: trabecular thickness; Tb.N: trabecular number; D.A: degree of anisotropy; STIFF: trabecular stiffness; PVM: peak Von-Mises stress; and SVM: skewness of Von-Mises stress histogram. presence or absence of recovery in BV/TV during reambulation. For those mice that showed recovery during reambulation $(\mathrm{n}=231$, $66 \%$ of the population), correlations between BV/TV and simulated mechanical parameters resembled those involving the entire population; strong at baseline and weakening progressively with unloading and reambulation (Table 5). Mice that experienced further deterioration of $\mathrm{BV} / \mathrm{TV}$ during reambulation ( $\mathrm{n}=117,34 \%$ of the population) demonstrated morphologic-mechanical associations that were similar to the entire F2 population at baseline and during unloading, but $\mathrm{R}^{2}$ values computed for changes during reambulation were smaller than in mice that had recovered BV/TV (Table 5). For both subpopulations, BV/TV and $\mathrm{Tb}$.Th were the variables that were most highly associated with trabecular bone's simulated mechanical properties.

\section{Discussion}

Changes in trabecular bone's simulated mechanical properties were contrasted with changes in its morphological indices in a genetically heterogeneous mouse population that was exposed to mechanical unloading and subsequent reambulation. At baseline, trabecular morphology was strongly associated with mechanical properties. The deterioration of trabecular bone volume fraction during unloading was paralleled by the demise of its mechanical properties as indicated by increased peak stresses and skewness in the stress distributions. During reambulation, one in three F2 mice experienced continued deterioration in trabecular bone volume fraction and architectural indices, except for trabecular thickness, continued to decline for the majority of the population. Conversely, most of the experimental mice showed at least some degree of recovery from unloading induced deterioration in peak stresses and stress distributions during reambulation. Mice that continued to lose bone volume fraction during reambulation had different trabecular morphology at baseline than those that recovered tissue

Table 4

Coefficients of determination $\left(\mathrm{R}^{2}\right)$ between morphological indices and mechanical properties at baseline and during unloading (\% changes) and during reambulation (\% changes).

\begin{tabular}{lllrl}
\hline & $\mathrm{R}^{2}$ & STIFF & PVM & SVM \\
\hline Baseline $(\mathrm{n}=436)$ & BV/TV & $88 \%$ & $66 \%$ & $44 \%$ \\
& Conn.D & $46 \%$ & $27 \%$ & $15 \%$ \\
& Tb.N & $69 \%$ & $50 \%$ & $28 \%$ \\
& Tb.Th & $70 \%$ & $73 \%$ & $48 \%$ \\
& D.A & $19 \%$ & $9 \%$ & $31 \%$ \\
Unloading $(\mathrm{n}=359)$ & BV/TV & $78 \%$ & $46 \%$ & $20 \%$ \\
& Conn.D & $27 \%$ & $9 \%$ & $1 \%$ \\
& Tb.N & $46 \%$ & $28 \%$ & $13 \%$ \\
& Tb.Th & $65 \%$ & $50 \%$ & $25 \%$ \\
& D.A & $14 \%$ & $2 \%$ & $5 \%$ \\
& BV/TV & $55 \%$ & $24 \%$ & $17 \%$ \\
& Conn.D & $16 \%$ & $6 \%$ & $3 \%$ \\
& Tb.N & $6 \%$ & $11 \%$ & $n .5$. \\
& Tb.Th & $38 \%$ & $20 \%$ & $15 \%$ \\
& D.A & $14 \%$ & $2 \%$ & $11 \%$ \\
\hline
\end{tabular}

All correlations were positive for trabecular stiffness (STIFF) and negative for peak (PVM) and skewness (SVM) of Von Mises stress distributions. $\mathrm{p}<0.05$ unless noted. n.s.: not significant, $\mathrm{p}>0.05$. 
Table 5

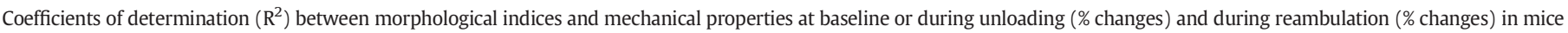
that showed either positive recovery $(n=231)$ or further deterioration $(n=117)$ during reambulation.

\begin{tabular}{|c|c|c|c|c|c|c|c|c|c|c|}
\hline & & \multicolumn{3}{|c|}{ Baseline } & \multicolumn{3}{|c|}{ Unloading } & \multicolumn{3}{|c|}{ Reambulation } \\
\hline & & STIFF & PVM & SVM & STIFF & PVM & SVM & STIFF & PVM & SVM \\
\hline \multirow[t]{5}{*}{ Positive recovery $(n=231)$} & $\mathrm{BV} / \mathrm{TV}$ & $88 \%$ & $69 \%$ & $39 \%$ & $73 \%$ & $42 \%$ & $20 \%$ & $47 \%$ & $9 \%$ & $15 \%$ \\
\hline & Conn.D & $45 \%$ & $33 \%$ & $12 \%$ & $27 \%$ & $8 \%$ & n.s. & $10 \%$ & $3 \%$ & $2 \%$ \\
\hline & Tb.N & $68 \%$ & $52 \%$ & $26 \%$ & $38 \%$ & $23 \%$ & $9 \%$ & n.s. & $5 \%$ & n.s. \\
\hline & Tb.Th & $71 \%$ & $71 \%$ & $45 \%$ & $61 \%$ & $47 \%$ & $27 \%$ & $30 \%$ & $7 \%$ & $9 \%$ \\
\hline & D.A & $21 \%$ & $9 \%$ & $30 \%$ & $15 \%$ & n.s. & $4 \%$ & $14 \%$ & n.s. & $12 \%$ \\
\hline \multirow[t]{5}{*}{ Further deterioration $(\mathrm{n}=117$ ) } & $\mathrm{BV} / \mathrm{TV}$ & $97 \%$ & $61 \%$ & $50 \%$ & $82 \%$ & $47 \%$ & $15 \%$ & $6 \%$ & $11 \%$ & ns \\
\hline & Conn.D & $44 \%$ & $22 \%$ & $19 \%$ & $26 \%$ & $7 \%$ & n.s. & n.s. & n.s. & n.s. \\
\hline & Tb.N & $66 \%$ & $44 \%$ & $33 \%$ & $54 \%$ & $31 \%$ & $16 \%$ & n.s. & n.s. & $8 \%$ \\
\hline & Tb.Th & $69 \%$ & $74 \%$ & $51 \%$ & $70 \%$ & $51 \%$ & $19 \%$ & $10 \%$ & $14 \%$ & $5 \%$ \\
\hline & D.A & $15 \%$ & $6 \%$ & $22 \%$ & $14 \%$ & $9 \%$ & $7 \%$ & n.s. & n.s. & n.s. \\
\hline
\end{tabular}

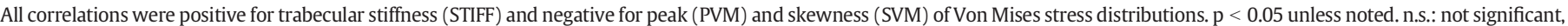
$\mathrm{p}>0.05$.

during reambulation. Morphologic-mechanical associations, while strong at baseline, weakened during unloading and reambulation, indicating an uncoupling between trabecular bone's morphological and mechanical properties. Overall, our results demonstrate a great disparity of mechano-responses across a genetically heterogeneous mouse population with a recovery from unloading induced trabecular corrosion that predominantly comprises trabecular thickness and mechanical properties but not other aspects of trabecular architecture.

FE models are powerful tools for describing stress/strain distributions in complex structures such as trabecular bone and provide more accurate estimates for the risk of failure than the use of imaging modalities alone [39-41]. However, limitations of FE models need to be considered. Data output can be highly dependent on loading and material assumptions $[42,43]$, a concern that was addressed here by using a simple uni-axial compression test and a linear elastic solution that reduces the influence of material properties as only relative differences within and between groups were considered. Our FE model also did not produce important data on fracture risk that is routinely generated with invasive mechanical testing procedures such as post-yield properties. However, the longitudinal design of our study necessitated the use of FE models over invasive testing of trabecular bone mechanical properties, a test that is difficult to perform in murine models. Inherently, the study design precluded all invasive measurement including the characterization of material properties known to play a role in bone mechanics such as microdamage accumulation, mineralization, or collagen cross-linking. This study focused on trabecular bone which in this mouse population is more sensitive to altered mechanical demand than cortical bone [30]. Even though we have previously reported a lack of co-adaptation between cortical and trabecular bone during unloading/reambulation in this experiment [30], it is clear that future studies that aim to estimate overall fracture risk will need to integrate cortical bone.

Using a genetically heterogeneous mouse population and exposing them to varying levels of weight bearing allowed us to observe large differences in morphologic and mechanical changes between individual mice. As expected from earlier studies, mechanical properties reflected this variability in morphology with strong associations in normally ambulating mice $[40,44,45]$. Mechanical unloading not only induced morphological deterioration and increased risk of mechanical failure in the trabecular structure $[46,47]$, but also weakened the coupling between morphological and mechanical parameters. Even though the associations were not as strong, variations in longitudinal changes in bone volume fraction and trabecular thickness were able to moderately explain variations in longitudinal changes in stiffness, peak stresses and stress distributions. Previous human space flight studies described a lack of association between loss in mechanical strength and bone mass analyzed by DXA [4]. Our results on the other hand suggest that at least some morphological parameters may remain linked to mechanical properties during the application of catabolic mechanical signals. This difference may be accounted for by differences in imaging modalities as $\mu \mathrm{CT}$ based imaging, but not DXA, can describe the architecture of the structure.

During reambulation, associations between changes in morphological and mechanical properties were weak. Regardless, trabecular thickness and bone volume fraction remained the strongest predictors of bone's mechanical properties. Thus, the recovery of trabecular bone's mechanical variables during reambulation may be, at least in part, uncoupled from morphological changes. While the increase in trabecular bone volume fraction was small during reambulation, the resorption of trabecular struts and their connections was mechanically compensated for by thickening of the remaining trabecular struts. Inherently, the addition of trabecular tissue can increase the stiffness of the structure and reduce peak stresses but these data also demonstrate that increased bone volume fraction by itself cannot determine the efficiency of the structure to bear load. The much greater percentage of mice recovering their mechanical, rather than their morphological, properties stresses the importance of assessing bone structural integrity during skeletal recovery.

A likely mechanism for reambulation induced changes in trabecular morphology may lie with the adaptive behavior of bone tissue. Upon reambulation, trabecular struts were reloaded but due to the extensive loss of tissue, load bearing characteristics of the trabecular structure were altered significantly, causing the presence of over- and underloaded struts. Overloaded structures are at risk of mechanical failure or microdamage $[48,49]$ providing signals for trabeculae to thicken during reambulation $[50,51]$. This response is not limited to recovery from a catabolic state but may simply present a response to large loading increases [52]. Our data demonstrate that during reambulation it is possible for a trabecular structure to enhance its mechanical integrity through a net decrease in peak stress and skewness values at the same time when BV/TV continues to decrease.

Similar losses and thickening of individual struts were observed in weight-bearing bones of mice [53] and rats [54] under strong catabolic signals such as OVX or aging. Furthermore, trabeculae that are thicker than normal tend to align with the primary loading direction, indicating a mechanically adaptive process in these structures [54]. We found more uniform load distributions with smaller peak stresses and an average increase in trabecular thickness but not trabecular number, suggesting an adaptive response of the remaining struts in the principal (longitudinal) loading direction. Even though this adaptation is beneficial for loads applied during regular weight-bearing, it does not protect the structure from transverse or torsional loads. Because individuals who experience osteoporotic fractures have weaker trabecular structures in the transverse loading direction [55], in spite of similar load bearing characteristics in the longitudinal direction [56], the risk of fracture may be greater in our mice than a simulated uniaxial mechanical test suggests. While emphasizing the importance of determining mechanical properties of trabecular bone, our data also suggest that 
after exposure to altered mechanical environments, estimating individual risk of mechanical failure in trabecular bone may need to include the consideration of loading direction.

In summary, mechanical unloading induced mechanical deterioration of trabecular bone that was highly variable in genetically heterogeneous mice. Recovery of trabecular tissue was much more limited than the recovery of mechanical properties which reduced stress magnitudes and increased efficiency of micro-mechanical load bearing. These mechanical benefits were modulated by thickening of trabecular struts even while under-connected struts continued to resorb. When extrapolated to astronauts or individuals recovering from bedrest, our data indicate that even 3D imaging modalities such as QCT may overestimate the risk of trabecular fracture based on scans taken during the recovery phase and that FE modeling may aid in the identification of those individuals with trabecular structures susceptible to failure.

\section{Acknowledgments}

Financial support by NASA (NAG 9-1499, NNX08BA35G and NNX12AL25G) is gratefully acknowledged.

\section{Conflict of interest}

All authors have no conflicts of interest.

\section{Appendix A. Supplementary data}

Supplementary data to this article can be found online at http://dx. doi.org/10.1016/j.bone.2014.05.009.

\section{References}

[1] Ozcivici E, Luu YK, Adler B, Qin YX, Rubin J, Judex S, et al. Mechanical signals as anabolic agents in bone. Nat Rev Rheumatol 2010;6:50-9.

[2] Lang T, LeBlanc A, Evans H, Lu Y, Genant H, Yu A. Cortical and trabecular bone mineral loss from the spine and hip in long-duration spaceflight. J Bone Miner Res 2004;19:1006-12

[3] Orwoll ES, Adler RA, Amin S, Binkley N, Lewiecki EM, Petak SM, et al. Skeletal health in long-duration astronauts: nature, assessment, and management recommendations from the NASA bone summit. J Bone Miner Res 2013;28:1243-55.

[4] Keyak JH, Koyama AK, LeBlanc A, Lu Y, Lang TF. Reduction in proximal femoral strength due to long-duration spaceflight. Bone 2009;44:449-53.

[5] Sun LW, Fan YB, Li DY, Zhao F, Xie T, Yang X, et al. Evaluation of the mechanical properties of rat bone under simulated microgravity using nanoindentation. Acta Biomater 2009;5:3506-11.

[6] LeBlanc A, Lin C, Shackelford L, Sinitsyn V, Evans H, Belichenko O, et al. Muscle volume, MRI relaxation times (T2), and body composition after spaceflight. J Appl Physiol 2000;89:2158-64.

[7] Sun B, Cao XS, Zhang LF, Liu C, Ni HY, Cheng JH, et al. Daily 4-h head-up tilt is effective in preventing muscle but not bone atrophy due to simulated microgravity. J Gravit Physiol 2003;10:29-38.

[8] Muir J, Judex S, Qin YX, Rubin C. Postural instability caused by extended bed rest is alleviated by brief daily exposure to low magnitude mechanical signals. Gait Posture 2011;33:429-35.

[9] Ziambaras K, Civitelli R, Papavasiliou SS. Weightlessness and skeleton homeostasis. Hormones (Athens) 2005;4:18-27.

[10] Narici MV, de Boer MD. Disuse of the musculo-skeletal system in space and on earth. Eur J Appl Physiol 2011;111:403-20.

[11] Morey-Holton E, Globus RK, Kaplansky A, Durnova G. The hindlimb unloading rat model: literature overview, technique update and comparison with space flight data. Adv Space Biol Med 2005;10:7-40.

[12] Swift JM, Nilsson MI, Hogan HA, Sumner LR, Bloomfield SA. Simulated resistance training during hindlimb unloading abolishes disuse bone loss and maintains muscle strength. J Bone Miner Res 2010;25:564-74.

[13] Rubin C, Xu G, Judex S. The anabolic activity of bone tissue, suppressed by disuse, is normalized by brief exposure to extremely low-magnitude mechanical stimuli. FASEB J 2001;15:2225-9.

[14] Ozcivici E, Garman R, Judex S. High-frequency oscillatory motions enhance the simulated mechanical properties of non-weight bearing trabecular bone. J Biomech 2007;40:3404-11.

[15] Ozcivici E, Luu YK, Rubin CT, Judex S. Low-level vibrations retain bone marrow's osteogenic potential and augment recovery of trabecular bone during reambulation. PLoS One 2010;5:e11178.

[16] Lang TF, LeBlanc AD, Evans HJ, Lu Y. Adaptation of the proximal femur to skeletal reloading after long-duration spaceflight. J Bone Miner Res 2006;21:1224-30.
[17] Sibonga JD, Evans HJ, Sung HG, Spector ER, Lang TF, Oganov VS, et al. Recovery of spaceflight-induced bone loss: bone mineral density after long-duration missions as fitted with an exponential function. Bone 2007;41:973-8.

[18] Carpenter RD, LeBlanc AD, Evans H, Sibonga JD, Lang TF. Long-term changes in the density and structure of the human hip and spine after long-duration spaceflight Acta Astronaut 2010;67:71-81.

[19] Vico L, Collet P, Guignandon A, Lafage-Proust MH, Thomas T, Rehaillia M, et al. Effects of long-term microgravity exposure on cancellous and cortical weight-bearing bones of cosmonauts. Lancet 2000;355:1607-11.

[20] Allen MR, Hogan HA, Bloomfield SA. Differential bone and muscle recovery following hindlimb unloading in skeletally mature male rats. J Musculoskelet Neuronal Interact 2006;6:217-25.

[21] Keaveny TM. Biomechanical computed tomography-noninvasive bone strength analysis using clinical computed tomography scans. Ann N Y Acad Sci 2010;1192:57-65.

[22] Cody DD, Gross GJ, Hou FJ, Spencer HJ, Goldstein SA, Fyhrie DP. Femoral strength is better predicted by finite element models than QCT and DXA. J Biomech 1999;32:1013-20.

[23] Orwoll ES, Marshall LM, Nielson CM, Cummings SR, Lapidus J, Cauley JA, et al. Finite element analysis of the proximal femur and hip fracture risk in older men. J Bone Miner Res 2009;24:475-83.

[24] Keyak JH, Sigurdsson S, Karlsdottir G, Oskarsdottir D, Sigmarsdottir A, Zhao S, et al. Male-female differences in the association between incident hip fracture and proximal femoral strength: a finite element analysis study. Bone 2011;48:1239-45.

[25] Fields AJ, Lee GL, Liu XS, Jekir MG, Guo XE, Keaveny TM. Influence of vertical trabeculae on the compressive strength of the human vertebra. J Bone Miner Res 2011;26:263-9

[26] Judex S, Boyd S, Qin YX, Turner S, Ye K, Muller R, et al. Adaptations of trabecular bone to low magnitude vibrations result in more uniform stress and strain under load. Ann Biomed Eng 2003;31:12-20.

[27] Hasegawa Y, Schneider P, Reiners C. Age, sex, and grip strength determine architectural bone parameters assessed by peripheral quantitative computed tomography (pQCT) at the human radius. J Biomech 2001;34:497-503.

[28] LeBlanc AD, Spector ER, Evans HJ, Sibonga JD. Skeletal responses to space flight and the bed rest analog: a review. J Musculoskelet Neuronal Interact 2007;7:33-47.

[29] Shirazi-Fard Y, Kupke JS, Bloomfield SA, Hogan HA. Discordant recovery of bone mass and mechanical properties during prolonged recovery from disuse. Bone 2013;52:433-43

[30] Judex S, Zhang W, Donahue LR, Ozcivici E. Genetic loci that control the loss and regain of trabecular bone during unloading and reambulation. J Bone Miner Res 2013;28:1537-49.

[31] Ozcivici E, Zhang W, Donahue LR, Judex S. Quantitative trait loci that modulate trabecular bone's risk of failure during unloading and reloading. Bone 2014;64:25-32.

[32] Judex S, Garman R, Squire M, Busa B, Donahue LR, Rubin C. Genetically linked sitespecificity of disuse osteoporosis. J Bone Miner Res 2004;19:607-13.

[33] Morey-Holton E, Wronski TJ. Animal models for simulating weightlessness. Physiologist 1981;24:545-8.

[34] Lublinsky S, Ozcivici E, Judex S. An automated algorithm to detect the trabecularcortical bone interface in micro-computed tomographic images. Calcif Tissue Int 2007;81:285-93.

[35] Busa B, Miller LM, Rubin CT, Qin YX, Judex S. Rapid establishment of chemical and mechanical properties during lamellar bone formation. Calcif Tissue Int 2005;77:386-94.

[36] Rho JY, Tsui TY, Pharr GM. Elastic properties of human cortical and trabecular lamellar bone measured by nanoindentation. Biomaterials 1997:18:1325-30.

[37] Bayraktar HH, Morgan EF, Niebur GL, Morris GE, Wong EK, Keaveny TM. Comparison of the elastic and yield properties of human femoral trabecular and cortical bone tissue. J Biomech 2004;37:27-35.

[38] Van Rietbergen B, Weinans H, Huiskes R, Odgaard A. A new method to determine trabecular bone elastic properties and loading using micromechanical finiteelement models. J Biomech 1995;28:69-81.

[39] Van RB, Huiskes R, Eckstein F, Ruegsegger P. Trabecular bone tissue strains in the healthy and osteoporotic human femur. J Bone Miner Res 2003;18:1781-8.

[40] Van Der Linden JC, Birkenhager-Frenkel DH, Verhaar JA, Weinans H. Trabecular bone's mechanical properties are affected by its non-uniform mineral distribution. J Biomech 2001;34:1573-80.

[41] Shi X, Liu XS, Wang X, Guo XE, Niebur GL. Type and orientation of yielded trabeculae during overloading of trabecular bone along orthogonal directions. J Biomech 2010;43:2460-6.

[42] Grine FE, Judex S, Daegling DJ, Ozcivici E, Ungar PS, Teaford MF, et al. Craniofacial biomechanics and functional and dietary inferences in hominin paleontology. J Hum Evol 2010;58:293-308.

[43] Daegling DJ, Judex S, Ozcivici E, Ravosa MJ, Taylor AB, Grine FE, et al. Viewpoints: feeding mechanics, diet, and dietary adaptations in early hominins. Am J Phys Anthropol 2013;151:356-71.

[44] Morgan EF, Bayraktar HH, Yeh OC, Majumdar S, Burghardt A, Keaveny TM. Contribution of inter-site variations in architecture to trabecular bone apparent yield strains. J Biomech 2004;37:1413-20.

[45] Bevill G, Eswaran SK, Gupta A, Papadopoulos P, Keaveny TM. Influence of bone volume fraction and architecture on computed large-deformation failure mechanisms in human trabecular bone. Bone 2006;39:1218-25.

[46] Bozic KJ, Keyak JH, Skinner HB, Bueff HU, Bradford DS. Three-dimensional finite element modeling of a cervical vertebra: an investigation of burst fracture mechanism. J Spinal Disord 1994;7:102-10.

[47] Niebur GL, Feldstein MJ, Yuen JC, Chen TJ, Keaveny TM. High-resolution finite element models with tissue strength asymmetry accurately predict failure of trabecular bone. J Biomech 2000;33:1575-83. 
[48] Silva MJ, Keaveny TM, Hayes WC. Computed tomography-based finite element analysis predicts failure loads and fracture patterns for vertebral sections. J Orthop Res 1998;16:300-8.

[49] Nagaraja S, Couse TL, Guldberg RE. Trabecular bone microdamage and microstructural stresses under uniaxial compression. J Biomech 2005;38:707-16.

[50] Huiskes R, Ruimerman R, van Lenthe GH, Janssen JD. Effects of mechanical forces on maintenance and adaptation of form in trabecular bone. Nature 2000;405:704-6.

[51] Mori S, Burr DB. Increased intracortical remodeling following fatigue damage. Bone 1993;14:103-9.

[52] Lambers FM, Schulte FA, Kuhn G, Webster DJ, Muller R. Mouse tail vertebrae adapt to cyclic mechanical loading by increasing bone formation rate and decreasing bone resorption rate as shown by time-lapsed in vivo imaging of dynamic bone morphometry. Bone 2011;49:1340-50.
[53] Klinck J, Boyd SK. The magnitude and rate of bone loss in ovariectomized mice differs among inbred strains as determined by longitudinal in vivo microcomputed tomography. Calcif Tissue Int 2008;83:70-9.

[54] Waarsing JH, Day JS, Verhaar JA, Ederveen AG, Weinans H. Bone loss dynamics result in trabecular alignment in aging and ovariectomized rats. J Orthop Res 2006;24:926-35.

[55] Homminga J, McCreadie BR, Ciarelli TE, Weinans H, Goldstein SA, Huiskes R. Cancellous bone mechanical properties from normals and patients with hip fractures differ on the structure level, not on the bone hard tissue level. Bone 2002;30:759-64.

[56] Homminga J, Van-Rietbergen B, Lochmuller EM, Weinans H, Eckstein F, Huiskes R. The osteoporotic vertebral structure is well adapted to the loads of daily life, but not to infrequent "error" loads. Bone 2004;34:510-6. 